\title{
Modelling wildland fire propagation by tracking random fronts
}

\author{
G. Pagnini ${ }^{1,2}$ and A. Mentrelli ${ }^{1,3}$ \\ ${ }^{1}$ BCAM - Basque Center for Applied Mathematics, Alameda de Mazarredo 14, 48009 Bilbao, Basque Country, Spain \\ ${ }^{2}$ Ikerbasque, Basque Foundation for Science, Alameda Urquijo 36-5, Plaza Bizkaia, 48011 Bilbao, Basque Country, Spain \\ ${ }^{3}$ Department of Mathematics, University of Bologna, Piazza di Porta San Donato 5, 40126 Bologna, Italy
}

Correspondence to: G. Pagnini (gpagnini@bcamath.org)

Received: 29 September 2013 - Published in Nat. Hazards Earth Syst. Sci. Discuss.: 19 November 2013

Revised: 22 May 2014 - Accepted: 26 June 2014 - Published: 28 August 2014

\begin{abstract}
Wildland fire propagation is studied in the literature by two alternative approaches, namely the reactiondiffusion equation and the level-set method. These two approaches are considered alternatives to each other because the solution of the reaction-diffusion equation is generally a continuous smooth function that has an exponential decay, and it is not zero in an infinite domain, while the levelset method, which is a front tracking technique, generates a sharp function that is not zero inside a compact domain. However, these two approaches can indeed be considered complementary and reconciled. Turbulent hot-air transport and fire spotting are phenomena with a random nature and they are extremely important in wildland fire propagation. Consequently, the fire front gets a random character, too; hence, a tracking method for random fronts is needed. In particular, the level-set contour is randomised here according to the probability density function of the interface particle displacement. Actually, when the level-set method is developed for tracking a front interface with a random motion, the resulting averaged process emerges to be governed by an evolution equation of the reaction-diffusion type. In this reconciled approach, the rate of spread of the fire keeps the same key and characterising role that is typical of the level-set approach. The resulting model emerges to be suitable for simulating effects due to turbulent convection, such as fire flank and backing fire, the faster fire spread being because of the actions by hot-air pre-heating and by ember landing, and also due to the fire overcoming a fire-break zone, which is a case not resolved by models based on the level-set method. Moreover, from the proposed formulation, a correction follows for the formula of the rate of spread which is due to the mean
\end{abstract}

jump length of firebrands in the downwind direction for the leeward sector of the fireline contour. The presented study constitutes a proof of concept, and it needs to be subjected to a future validation.

\section{Introduction}

Modelling wildland fire propagation is a twofold challenging task because it is motivated by social and scientific reasons. In fact, from the social point of view, fire is a hazardous phenomenon for human safety and property and also for ecosystems, because it can cause disruption and is an important source of pollutants (Strada et al., 2012). Moreover, it is a challenging task for scientific reasons because it is a complex phenomenon involving multi-physics and multiscale processes, and it is affected by nonlinear interactions with other Earth processes (Viegas, 1998).

Two different approaches are mainly adopted in the literature to investigate wildland fire propagation. One of these modelling approaches is based on evolution equations of the reaction-diffusion type (e.g. Weber et al., 1997; Asensio and Ferragut, 2002; Mandel et al., 2008; Babak et al., 2009), and the other is based on the front tracking technique named the level-set method (Sethian and Smereka, 2003): see for example Mallet et al. (2009), Rehm and McDermott (2009), and Mandel et al. (2011).

In a broad sense, diffusion processes are named those small-scale stochastic processes whose displacement on large scales is governed by a master equation. Diffusion processes are generally driven by parabolic equations, although 
hyperbolic equations are just as good or even better for modelling diffusive processes because of the finite front velocity, e.g. the telegraph equation. When a source term is added, the resulting equation is termed a reaction-diffusion equation. Hence, reaction-diffusion equations model the propagation of a reacting interface embedded in a random environment. This type of equation can embody a very general mathematical model that can be applied to several phenomena.

In general, the level-set method is particularly useful for handling problems in which the speed of an evolving interface is dependent on interface properties such as curvature and normal direction, as well as on the boundary conditions at the interface location. Hence, it is suitable for problems in which the topology of the evolving interface changes during the events and for problems in which sharp corners and cusps can be generated (Sethian and Smereka, 2003).

These approaches are considered alternatives to each other because of the different behaviours of their solutions. In particular, the solution of the reaction-diffusion equation is generally a continuous smooth function that has an exponential decay, and then it is not zero on an infinite domain, while the solution obtained by the level-set method is a sharp function that is zero outside a compact domain. However, these two approaches can indeed be considered complementary and can be reconciled.

In fact, extremely important phenomena in wildland fire propagation are turbulent hot-air transport due to the turbulent nature of the atmospheric boundary layer that can consequently affect fire-atmosphere interactions (Clark et al., 1996; Potter, 2002, 2012a, b; Linn and Cunningham, 2005; Cunningham and Linn, 2007; Sun et al., 2006; Clements et al., 2008; Filippi et al., 2009, 2011, 2013; Sun et al., 2009; Mandel et al., 2011; Forthofer and Goodrick, 2011), as well as the fire spotting phenomenon (Sardoy et al., 2007, 2008; Kortas et al., 2009; Perryman, 2009; Bhutia et al., 2010; Koo et al., 2010; Wang, 2011; Morgante, 2011; Perryman et al., 2013). Both processes have a random character; therefore, the fire front motion turns out to be random. Different stochastic approaches have been proposed in the literature (see e.g. Favier, 2004; Hunt, 2007; Boychuk et al., 2009; Almeida and Macau, 2011; Perryman et al., 2013).

Here, the level-set method for tracking fronts is extended to track random fronts. The frontline motion, whose propagation is determined by the rate of spread (ROS), is randomised by adding noise-generated random turbulent transport and fire spotting. The resulting averaged process emerges to be governed by an evolution equation of the reaction-diffusion type, and the ROS drives the source term. Actually, the randomisation of the fireline contour is performed according to the probability density function (PDF) of the front particle displacement, and the ROS of the fire keeps the same key and characterising role that is typical of the level-set approach. When the random motion turns out to be deterministic, the reaction-diffusion equation reduces to the Hamilton-Jacobi equation typical of the level-set method.
It should be stressed that, in the proposed approach, the randomisation of the fireline motion is accounted for as being due to physical processes, namely the turbulent hot-air transport and the fire spotting phenomenon. If uncertainties in the input data necessary for computing the ROS are to be taken into account, resulting in an ROS treated as a random variable, the model proposed here could be improved by coupling it with a data assimilation algorithm based, for example, on the so-called ensemble Kalman filter (Mandel et al., 2008; Beezley and Mandel, 2008; Cobb and Beezley, 2011; Rochoux et al., 2012, 2013, 2014a, b).

The present model emerges to be characterised by three major features:

- it is suitable for simulating the effects due to turbulent convection, such as flanking and backing fires, and the fire overcoming a fire-break zone, which is a case not resolved by models based on the level-set method;

- it accounts for the additional phenomenon of fire spotting, consisting in the ejection of embers that can potentially enhance the fire spread;

- it also accounts for pre-heating effects by hot air, resulting in a further enhancement of the fire spread.

Ejection of embers is modelled as an inherently random process with a known distribution of landing distances from the fireline. An additional term to the ROS is derived to include properly the effects of the mean jump length of firebrands.

Moreover, since the solution of the reaction-diffusion equation is not zero in an infinite domain, the potential fire ahead of the selected frontline can be considered to be a longrange action of the fire itself that then generates a pre-heating effect. In particular, the accumulation in time of such potential fire can be associated with an amount of heat and then related to the increasing of the fuel temperature (possibly up to the ignition threshold). Ignition is thus modelled as the consequence of sufficiently prolonged exposure to high temperatures. This accumulation can be regarded as a memory effect governed by the dynamics of the process that, clearly, cannot be dealt with by adding a suitable term to the ROS, which only allows local effects to be taken into account.

The paper is organised as follows. In Sect. 2, the approaches based on the reaction-diffusion equation and the level-set method for wildland fire propagation are discussed briefly. In Sect. 3, a picture to model wildland fire propagation is depicted and the mathematical formulation of a method for tracking random fronts is introduced. In Sect. 4 the proposed model is discussed and in Sect. 5 results from numerical simulations are shown. Finally, in Sect. 6, conclusions are reported. 


\section{Reaction-diffusion equations and level-set method in wildland fire propagation}

\subsection{Reaction-diffusion equation modelling}

An important observable for fire mapping is the temperature field. Actually, temperature is spread by molecular processes and turbulent flows, so it has a random character and is modelled by a diffusion process. Furthermore, the fire is an energy source, and a reaction-diffusion equation follows from conservation of energy and fuel on the basis of the combustion wave approach (Weber et al., 1997). Two-equation models concerning the average temperature field $T(\boldsymbol{x}, t)$ and the fuel mass fraction $Y(\boldsymbol{x}, t), Y \in[0,1]$, have been developed and analysed in the literature (see e.g. Montenegro et al., 1997; Asensio and Ferragut, 2002; Serón et al., 2005; Mandel et al., 2008; Babak et al., 2009). In a highly simplified form, these models read

$$
\begin{aligned}
& \frac{\partial T}{\partial t}+\boldsymbol{U} \nabla T=K \nabla^{2} T+\frac{Q}{c_{\mathrm{p}}} R Y-\frac{h A}{\rho c_{\mathrm{p}} V}\left(T-T_{\mathrm{a}}\right), \\
& \frac{\partial Y}{\partial t}=-R Y, \quad T>T_{\mathrm{a}},
\end{aligned}
$$

where $\boldsymbol{U}$ is the mean wind velocity, $K$ the diffusion coefficient, $Q$ the heat of reaction, $c_{\mathrm{p}}$ the specific heat of fuel, $R$ the reaction rate, $h$ the heat transfer coefficient from fuel to surroundings, $\rho$ the density of fuel, $A / V$ the surface area to volume ratio for fuel configuration and $T_{\mathrm{a}}$ the ambient temperature. This approach has also been calibrated, evaluated and implemented in a data assimilation system (Mandel et al., 2008). Further reaction-diffusion models for wildland fire propagation have been reviewed by Sullivan (2009).

However, in order to represent the burned/unburned front, reaction-diffusion equations have been developed whose solutions are sharp waves almost constant everywhere except in the interface region. Concerning this, since the levelset method (Sethian and Smereka, 2003), which is a front tracking technique, generates bi-value sharp solutions that are zero outside a compact domain, it emerges as the other widely used approach for modelling wildland fire propagation (Beezley et al., 2008; Rehm and McDermott, 2009; Mallet et al., 2009; Mandel et al., 2009; Dobrinkova et al., 2011; Mandel et al., 2011; Coen et al., 2013).

\subsection{General formulation of the level-set method}

The level-set method can be described briefly as follows. Let $\Gamma$ be a simple closed curve, or an ensemble of simple nonintersecting closed curves, representing a propagating interface in two dimensions, and let $\gamma: \mathcal{S} \times[0,+\infty[\rightarrow \mathbb{R}$ be a function defined on the domain of interest $\mathcal{S} \subseteq \mathbb{R}^{2}$ such that the level-set $\gamma_{*}$, i.e. $\gamma(\boldsymbol{x}, t)=\gamma_{*}$, coincides with the evolving front, i.e. $\Gamma(t)=\left\{\boldsymbol{x} \in \mathcal{S} \mid \gamma(\boldsymbol{x}, t)=\gamma_{*}\right\}$. In the case of $\Gamma$ being an ensemble of $n$ curves, the ensemble of the $n$ interfaces is considered to be an interface.
The evolution of the field $\gamma$ is governed by a HamiltonJacobi equation, which reads as follows:

$\frac{D \gamma}{D t}=\frac{\partial \gamma}{\partial t}+\frac{\mathrm{d} \boldsymbol{x}}{\mathrm{d} t} \cdot \nabla \gamma=0, \quad \gamma(\boldsymbol{x}, t=0)=\gamma_{0}(\boldsymbol{x})$,

where $\gamma_{0}$ is the initial field embedding the interface $\Gamma$ at $t=0$, $\Gamma_{0} \equiv \Gamma(t=0)$.

If the motion of the interface is directed towards the normal $\hat{\boldsymbol{n}}=-\nabla \gamma /\|\nabla \gamma\|$, i.e.

$\frac{\mathrm{d} \boldsymbol{x}}{\mathrm{d} t}=\boldsymbol{V}(\boldsymbol{x}, t)=\mathcal{V}(\boldsymbol{x}, t) \hat{\boldsymbol{n}}$,

then Eq. (2) becomes

$\frac{\partial \gamma}{\partial t}=\mathcal{V}(\boldsymbol{x}, t)\|\nabla \gamma\|$,

which is the ordinary level-set equation, and $\gamma(\boldsymbol{x}, t)$ can be named the level-set function.

\subsection{Application of the level-set method to the wildland fire propagation}

Within the formalism introduced in Sect. 2.2, the subsets of the domain $\mathcal{S}$ corresponding to the interface $\Gamma$ and to the region $\Omega$ enclosed by $\Gamma$ (that represent, respectively, the burn area and the fire perimeter) may be conveniently identified as the positive-valued regions selected by the two indicator functions $\mathcal{I}_{\Gamma}, \mathcal{I}_{\Omega}: \mathcal{S} \times[0,+\infty[\rightarrow\{0,1\}$ defined as follows:

$\mathcal{I}_{\Gamma}(\boldsymbol{x}, t)=\left\{\begin{array}{ll}1, & \text { if } \gamma(\boldsymbol{x}, t)=\gamma_{*} \\ 0, & \text { elsewhere }\end{array}\right.$,

and

$\mathcal{I}_{\Omega}(\boldsymbol{x}, t)= \begin{cases}1, & \text { if } \gamma(\boldsymbol{x}, t) \leq \gamma_{*} \\ 0, & \text { elsewhere }\end{cases}$

The indicator functions at time $t=0$, i.e. $\mathcal{I}_{\Gamma}(\boldsymbol{x}, t=0)$ and $\mathcal{I}_{\Omega}(\boldsymbol{x}, t=0)$, describing the initial topology of the fire, are indicated in the following as $\mathcal{I}_{\Gamma_{0}}(\boldsymbol{x})$ and $\mathcal{I}_{\Omega_{0}}(\boldsymbol{x})$, respectively.

In the case of a fireline $\Gamma$ made of more than one closed curve, the domain $\Omega$ is not simply connected, resulting in more than one burned area evolving independently.

When the application to wildland fire propagation is considered, the quantity $\mathcal{V}(\boldsymbol{x}, t)$, which has the dimension of a velocity, is identified by the ROS. The ROS value essentially depends on environmental conditions, i.e. the intensity and direction of the wind and the orography of the terrain, and on the fuel conditions, i.e. the type and characteristics of the vegetation. Several determinations of the ROS have been proposed in the literature; some are based on experimental data and others on certain physical insights (see e.g. Rothermel, 1972; Finney, 2002, 2003; Balbi et al., 2007, 2009; Mallet et al., 2009). 
Finally, instead of physically based differential equations, empirically observed properties of the fire such as the ROS can be used to model fireline evolution. In this regard, empirically or physically based formulae for the ROS can be straightforwardly included in the level-set method. Data assimilation (Mandel et al., 2009) has also been considered for the level-set approach, and it has been implemented in coupled weather-wildland fire models (Mandel et al., 2009, 2011; Coen et al., 2013) as well.

\section{Model picture and mathematical formulation of a method for tracking random fronts}

The approach derived in this section is an improvement of the approach originally formulated for a Lagrangian description of turbulent premixed combustion (Pagnini and Bonomi, 2011), and later extended to the study of wildland fire propagation, including the effects of turbulence (Pagnini and Massidda, 2012a, b). Here, the latter model is developed further in order to include fire spotting phenomena.

Let a large number of potential flame holders be distributed over the surface $\mathcal{S}$ covered by the fuel. Before the fire starts, each one of these potential flame holders stays at rest with a switched-off torch. When the fire starts, the torches of some potential flame holders are switched on, so that they turn into active flame holders; the locus of these initial active flame holders is the fireline $\Gamma_{0}$.

The active flame holders start to move with their burning torches. After a while, when an active flame holder reaches a potential flame holder, the latter turns into an $a c$ tive flame holder, too. As a consequence, the number of active flame holders and the length of the fireline $\Gamma$ increase in time. However, the growing process of the fireline length, $\mathcal{L}(t)$, and that of the number of active flame holders, $\mathcal{N}(t)$, are strongly dependent. In fact, when the length of the fireline grows, the number of active flame holders also increases, because the fireline contour can grow solely if a new potential flame holder turns into an active flame holder. To conclude, the growing ratio of the fireline, i.e. $\mathcal{L}(t) / \mathcal{L}(0)$, and that of the number of active flame holders, i.e. $\mathcal{N}(t) / \mathcal{N}(0)$, are equal. Hence, a constant action arc length $d=\mathcal{L}(t) / \mathcal{N}(t)=\mathcal{L}(0) / \mathcal{N}(0)$ can be associated with each active flame holder.

The above argument is based on the idea that active flame holders and constant action arc length can be compared with the concepts of Lagrangian markers and constant fireperimeter resolution introduced in the front tracking method discussed by Filippi et al. (2010, 2013).

Let the motion of each active flame holder be random, e.g. due to turbulence and fire spotting effects. For any realisation indexed by $\omega$, the random trajectory of each active flame holder is stated to be $\boldsymbol{X}^{\omega}\left(t, \overline{\boldsymbol{x}}_{0}\right)$, with the same fixed initial condition $\boldsymbol{X}^{\omega}\left(0, \overline{\boldsymbol{x}}_{0}\right)=\overline{\boldsymbol{x}}_{0}$ in all realisations.
By using statistical mechanics formalism (Klimontovich, 1994), the trajectory of a single active flame holder is marked out by the one-particle density function $f^{\omega}(\boldsymbol{x} ; t)=\delta\left(\boldsymbol{x}-\boldsymbol{X}^{\omega}\left(t, \overline{\boldsymbol{x}}_{0}\right)\right)$, where $\delta(\boldsymbol{x})$ is the Dirac $\delta$ function.

Observing that in the deterministic case the level-set function $\gamma$ solution of Eq. (4) may be written as

$\gamma(\boldsymbol{x}, t)=\int_{\mathcal{S}} \gamma(\overline{\boldsymbol{x}}, t) \delta(\boldsymbol{x}-\overline{\boldsymbol{x}}) d \overline{\boldsymbol{x}}$,

the effects of randomness are incorporated into the model, assuming that, in the $\omega$ realisation, the level-set function $\gamma^{\omega}$ embedding the fireline $\Gamma^{\omega}$ is obtained as a straightforward generalisation of Eq. (7) as follows:

$\gamma^{\omega}(\boldsymbol{x}, t)=\int_{\mathcal{S}} \gamma(\overline{\boldsymbol{x}}, t) \delta\left(\boldsymbol{x}-\boldsymbol{X}^{\omega}(t, \overline{\boldsymbol{x}})\right) d \overline{\boldsymbol{x}}$.

Accordingly, $\mathcal{I}_{\Gamma}$ and $\mathcal{I}_{\Omega}$ are replaced by the new indicator functions $\mathcal{I}_{\Gamma^{\omega}}, \mathcal{I}_{\Omega^{\omega}}: \mathcal{S} \times[0,+\infty[\rightarrow\{0,1\}$ defined as follows:

$$
\begin{aligned}
\mathcal{I}_{\Gamma^{\omega}}(\boldsymbol{x}, t) & =\int_{S} \mathcal{I}_{\Gamma_{0}}\left(\overline{\boldsymbol{x}}_{0}\right) \delta\left(\boldsymbol{x}-\boldsymbol{X}^{\omega}\left(t, \overline{\boldsymbol{x}}_{0}\right)\right) d \overline{\boldsymbol{x}}_{0} \\
& =\int_{\Gamma_{0}} \delta\left(\boldsymbol{x}-\boldsymbol{X}^{\omega}\left(t, \overline{\boldsymbol{x}}_{0}\right)\right) d \overline{\boldsymbol{x}}_{0} \\
& =\int_{\Gamma(t)} \delta\left(\boldsymbol{x}-\boldsymbol{X}^{\omega}(t, \overline{\boldsymbol{x}})\right) d \overline{\boldsymbol{x}}
\end{aligned}
$$

and

$$
\begin{aligned}
\mathcal{I}_{\Omega^{\omega}}(\boldsymbol{x}, t) & =\int_{\mathcal{S}} \mathcal{I}_{\Omega_{0}}\left(\overline{\boldsymbol{x}}_{0}\right) \delta\left(\boldsymbol{x}-\boldsymbol{X}^{\omega}\left(t, \overline{\boldsymbol{x}}_{0}\right)\right) d \overline{\boldsymbol{x}}_{0} \\
& =\int_{\Omega_{0}} \delta\left(\boldsymbol{x}-\boldsymbol{X}^{\omega}\left(t, \overline{\boldsymbol{x}}_{0}\right)\right) d \overline{\boldsymbol{x}}_{0} \\
& =\int_{\Omega(t)} \delta\left(\boldsymbol{x}-\boldsymbol{X}^{\omega}(t, \overline{\boldsymbol{x}})\right) d \overline{\boldsymbol{x}},
\end{aligned}
$$

where, for any fixed initial condition $\overline{\boldsymbol{x}}_{0}$, the evolution of the deterministic trajectory is noted by $\overline{\boldsymbol{x}}(t)$ and is uniquely obtained by a deterministic time-reversible map $\overline{\boldsymbol{x}}(t)=\mathcal{F}\left(t, \overline{\boldsymbol{x}}_{0}\right)$. Moreover, the assumption of a constant arc length of action implies a constant density of flame holders along the fireline, from which an incompressibility-like condition follows, and then $J=d \overline{\boldsymbol{x}}_{0} / d \overline{\boldsymbol{x}}=1$.

Hence, denoting the ensemble average by $\langle\cdot\rangle$, the effective indicator of the burned region, $\varphi_{\mathrm{e}}(\boldsymbol{x}, t): \mathcal{S} \times[0$, $+\infty[\rightarrow[0,1]$, may be defined as 


$$
\begin{aligned}
\varphi_{\mathrm{e}}(\boldsymbol{x}, t)=\left\langle\mathcal{I}_{\Omega^{\omega}(t)}\right\rangle & =\left\langle\int_{\Omega(t)} \delta\left(\boldsymbol{x}-\boldsymbol{X}^{\omega}(t, \overline{\boldsymbol{x}})\right) d \overline{\boldsymbol{x}}\right\rangle \\
& =\int_{\Omega(t)}\left\langle\delta\left(\boldsymbol{x}-\boldsymbol{X}^{\omega}(t, \overline{\boldsymbol{x}})\right)\right\rangle d \overline{\boldsymbol{x}} \\
& =\int_{\Omega(t)} f(\boldsymbol{x} ; t \mid \overline{\boldsymbol{x}}) d \overline{\boldsymbol{x}},
\end{aligned}
$$

where $f(\boldsymbol{x} ; t \mid \overline{\boldsymbol{x}})=\left\langle\delta\left(\boldsymbol{x}-\boldsymbol{X}^{\omega}(t, \overline{\boldsymbol{x}})\right)\right\rangle$ is the PDF of the displacement of the active flame holders around the average position $\overline{\boldsymbol{x}}$. Equation (11) was originally proposed to model the burned mass fraction in turbulent premixed combustion (Pagnini and Bonomi, 2011).

It should be noted that the effective indicator $\varphi_{\mathrm{e}}$ introduced here is not an indicator function in the classical sense. In fact, adopting the language of fuzzy logic, it is properly a membership function; its range is the compact interval $[0,1]$ rather than the discrete set $\{0,1\}$. Despite this, since the concept of probability which led to Eq. (11) should not be confused with the concept of degree of truth (typical of fuzzy logic), $\varphi_{\mathrm{e}}$ is classified as an indicator function instead of as a membership function.

Making use of the indicator function $\mathcal{I}_{\Omega}$, Eq. (11) can be written further as

$\varphi_{\mathrm{e}}(\boldsymbol{x}, t)=\int_{S} \mathcal{I}_{\Omega}(\overline{\boldsymbol{x}}, t) f(\boldsymbol{x} ; t \mid \overline{\boldsymbol{x}}) d \overline{\boldsymbol{x}}$.

It is worth noting that the deterministic trajectory $\bar{x}$ is the trajectory of a point belonging to the ordinary level-set contour with the same initial condition $\overline{\boldsymbol{x}}_{0}$. In the deterministic case, i.e. $\boldsymbol{X}^{\omega}(t, \overline{\boldsymbol{x}})=\overline{\boldsymbol{x}}$ for all realisations, it turns out that $f(\boldsymbol{x} ; t \mid \overline{\boldsymbol{x}})=\delta(\boldsymbol{x}-\overline{\boldsymbol{x}})$, and from Eq. (12) it is recovered as $\varphi_{\mathrm{e}}(\boldsymbol{x}, t)=\mathcal{I}_{\Omega(t)}$.

It may also be noted that Eq. (12) is remarkably close to the formulation found in smoothed-particle hydrodynamics (SPH) theory (Monaghan, 2005); nonetheless, in the present approach, the choice of the kernel function, i.e. the function that weights each contribution according to the distance from the point of interest, and that of the smoothing length, are removed because they straightforwardly follow from the PDF $f(\boldsymbol{x} ; t \mid \overline{\boldsymbol{x}})$.

Applying the Reynolds transport theorem to Eq. (11), the evolution equation of the effective indicator $\varphi_{\mathrm{e}}(\boldsymbol{x}, t)$ reads as (Pagnini and Bonomi, 2011)

$$
\frac{\partial \varphi_{\mathrm{e}}}{\partial t}=\int_{\Omega(t)} \frac{\partial f}{\partial t} d \overline{\boldsymbol{x}}+\int_{\Omega(t)} \nabla_{\bar{x}} \cdot[\boldsymbol{V}(\overline{\boldsymbol{x}}, t) f(\boldsymbol{x} ; t \mid \overline{\boldsymbol{x}})] d \overline{\boldsymbol{x}}
$$

Taking into account that $f(\boldsymbol{x} ; t \mid \overline{\boldsymbol{x}})$ satisfies the evolution equation

$$
\frac{\partial f}{\partial t}=\mathcal{E} f
$$

where $\mathcal{E}=\mathcal{E}(\boldsymbol{x})$ is a generic evolution operator not acting on $\overline{\boldsymbol{x}}$ and $t$, Eq. (13) can be written as

$$
\frac{\partial \varphi_{\mathrm{e}}}{\partial t}=\mathcal{E} \varphi_{\mathrm{e}}+\int_{\Omega(t)} \nabla_{\bar{x}} \cdot[\boldsymbol{V}(\overline{\boldsymbol{x}}, t) f(\boldsymbol{x} ; t \mid \overline{\boldsymbol{x}})] d \overline{\boldsymbol{x}} .
$$

To conclude, let $\kappa(\overline{\boldsymbol{x}}, t)$ be the mean front curvature defined by $\kappa(\overline{\boldsymbol{x}}, t)=\nabla_{\overline{\boldsymbol{x}}} \cdot \hat{\boldsymbol{n}} / 2$. Since the fireline velocity with magnitude given by the ROS is actually a function of the curvature, rather than the position, i.e. $\boldsymbol{V}=\boldsymbol{V}(\kappa, t) \equiv \mathcal{V}(\kappa, t) \hat{\boldsymbol{n}}$, the evolution equation of $\varphi_{\mathrm{e}}(\boldsymbol{x}, t)$ becomes

$$
\begin{aligned}
\frac{\partial \varphi_{\mathrm{e}}}{\partial t} & =\mathcal{E} \varphi_{\mathrm{e}}+\int_{\Omega(t)} \boldsymbol{V} \cdot \nabla_{\overline{\boldsymbol{x}}} f d \overline{\boldsymbol{x}} \\
& +\int_{\Omega(t)} f\left\{\frac{\partial \mathcal{V}}{\partial \kappa} \nabla_{\bar{x}} \kappa \cdot \hat{\boldsymbol{n}}+2 \mathcal{V}(\kappa, t) \kappa(\overline{\boldsymbol{x}}, t)\right\} d \overline{\boldsymbol{x}} .
\end{aligned}
$$

Equation (16) is a reaction-diffusion type equation that is associated with the level-set equation (Eq. 4). The fireline propagation is thus affected, in the present model, by the ROS, i.e. $\boldsymbol{V}(\overline{\boldsymbol{x}}, t)=\mathcal{V}(\boldsymbol{x}, t) \hat{\boldsymbol{n}}$, the mean front curvature, i.e. $\kappa(\overline{\boldsymbol{x}}, t)$, the turbulent dispersion, and the fire spotting phenomenon, both modelled by means of a single PDF, i.e. $f(\boldsymbol{x} ; t \mid \overline{\boldsymbol{x}})$.

It is emphasised here that this formulation holds for any determination of the ROS (see e.g. Rothermel, 1972; Finney, 2002, 2003; Balbi et al., 2007, 2009; Mallet et al., 2009). For a deterministic motion, i.e. when $f(\boldsymbol{x} ; t \mid \overline{\boldsymbol{x}})=\delta(\boldsymbol{x}-\overline{\boldsymbol{x}})$, Eq. (16) reduces to the ordinary level-set equation (Eq. 4) (Pagnini and Bonomi, 2011).

Since, as pointed out previously, the range of the effective indicator $\varphi_{\mathrm{e}}$ is the compact interval $[0,1]$, a criterion to mark the effective burned region $\Omega_{\mathrm{e}}$ has to be stated. The choice here is to mark as burned the region in which the effective indicator exceeds an arbitrarily fixed threshold value $\varphi_{\mathrm{e}}^{\text {th }}$, i.e. $\Omega_{\mathrm{e}}(\boldsymbol{x}, t)=\left\{\boldsymbol{x} \in \mathcal{S} \mid \varphi_{\mathrm{e}}(\boldsymbol{x}, t)>\varphi_{\mathrm{e}}^{\text {th }}\right\}$. However, besides this criterion, a further criterion associated with an ignition delay due to the pre-heating action of the hot air or to the landing of firebrands should be introduced. This ignition delay was previously considered as a heating-before-burning mechanism due to the hot air (Pagnini and Massidda, 2012a, b). Actually, it can be generalised to include fire spotting.

The ignition delay can be understood as an electrical resistance. Since the fuel can burn because of two pathways, i.e. hot-air heating and firebrand landing, the resistance analogy suggests that the resulting ignition delay can be approximatively computed as resistances acting in parallel. Hence, letting $\tau_{\mathrm{h}}$ and $\tau_{\mathrm{f}}$ be the ignition delay due to hot air and firebrands, respectively, the joint ignition delay $\tau$ is

$\frac{1}{\tau}=\frac{1}{\tau_{\mathrm{h}}}+\frac{1}{\tau_{\mathrm{f}}}=\frac{\tau_{\mathrm{h}}+\tau_{\mathrm{f}}}{\tau_{\mathrm{h}} \tau_{\mathrm{f}}}$.

Finally, the heating-before-burning mechanism is depicted as the persistence in time of the effective fire front, i.e. 
$\psi(\boldsymbol{x}, t)=\int_{0}^{t} \varphi_{\mathrm{e}}(\boldsymbol{x}, \eta) \frac{d \eta}{\tau}$,

where $\psi(\boldsymbol{x}, 0)=0$ corresponds to the unburned initial condition. The amount of heat is proportional to the increasing of the fuel temperature $T(\boldsymbol{x}, t)$, with $T(\boldsymbol{x}, 0)=T_{\mathrm{a}}(\boldsymbol{x})$; then,

$\psi(\boldsymbol{x}, t) \propto \frac{T(\boldsymbol{x}, t)-T_{\mathrm{a}}(\boldsymbol{x})}{T_{\mathrm{ign}}-T_{\mathrm{a}}(\boldsymbol{x})}, \quad T(\boldsymbol{x}, t) \leq T_{\mathrm{ign}}$,

where $T_{\text {ign }}$ is the ignition temperature. Since it holds $T_{\mathrm{a}} \ll T_{\text {ign }}$, Eq. (19) reduces to

$\psi(\boldsymbol{x}, t) \propto \frac{T(\boldsymbol{x}, t)}{T_{\mathrm{ign}}}$.

Hence, if for simplicity the proportionality in Eq. (19) is replaced by the equality, in points $x \in \Omega^{\prime}(t)$ such that $\psi(\boldsymbol{x}, t)=1$, the ignition occurs and fire goes on according to Eq. (12) by setting $\mathcal{I}_{\Omega^{\prime}}(\boldsymbol{x}, t)=1$.

To conclude, in this framework the temperature field emerges to be established by the following equation:

$\frac{\partial T(\boldsymbol{x}, t)}{\partial t}=\varphi_{\mathrm{e}}(\boldsymbol{x}, t) \frac{T_{\mathrm{ign}}-T_{\mathrm{a}}(\boldsymbol{x})}{\tau}, \quad T(\boldsymbol{x}, t) \leq T_{\mathrm{ign}}$,

where $T(\boldsymbol{x}, 0)=T_{\mathrm{a}}(\boldsymbol{x})$. If $T_{\mathrm{a}}(\boldsymbol{x})=T_{\mathrm{a}}$ is constant, after using Eq. (15), Eq. (21) becomes the following reaction-diffusiontype equation:

$\frac{\partial T}{\partial t}=\mathcal{E} T+\frac{T_{\mathrm{ign}}-T_{\mathrm{a}}}{\tau}\left\{\mathcal{I}_{\Omega_{0}}(\boldsymbol{x})+\mathcal{W}(\boldsymbol{x}, t)\right\}$,

where the identity $\varphi_{\mathrm{e}}(\boldsymbol{x}, 0)=\mathcal{I}_{\Omega_{0}}(\boldsymbol{x})$ is used, and

$\mathcal{W}(\boldsymbol{x}, t)=\int_{0}^{t}\left\{\int_{\Omega(\theta)} \nabla_{\bar{x}} \cdot[\boldsymbol{V}(\overline{\boldsymbol{x}}, \theta) f(\boldsymbol{x} ; \theta \mid \overline{\boldsymbol{x}})] d \overline{\boldsymbol{x}}\right\} d \theta$.

\section{Model discussion}

The random trajectory of each active flame holder is determined as $\boldsymbol{X}^{\omega}(t, \boldsymbol{x})=\overline{\boldsymbol{x}}_{\mathrm{ROS}}+\chi^{\omega}+\xi^{\omega}$, where $\overline{\boldsymbol{x}}_{\mathrm{ROS}}$ is a deterministic position driven by the ROS according to Eq. (3), and $\chi$ and $\xi$ are the contributions corresponding to randomly generated turbulence and fire spotting, respectively.

The instantaneous front velocity can then also be represented by the sum of a deterministic part and random contributions. This formulation has a formal analogy with the so-called ensemble Kalman filter (EnKF) (Mandel et al., 2008; Beezley and Mandel, 2008; Cobb and Beezley, 2011; Rochoux et al., 2012, 2013, 2014a, b). The EnKF is a statistical operational technique for handling uncertainties in the estimation of the ROS, but uncertainties in measurements are not straightforwardly related to physical random fluctuations, and data error is generally Gaussian distributed according to pure statistical arguments. In contrast, the proposed approach is based on the idea of considering fluctuations and their dynamics as being due to physical processes with a random nature. The present physical picture allows one to consider each involved process separately, and statistics of fluctuations are described by specific models. The PDF of fluctuations $f(\boldsymbol{x}$; $t \mid \bar{x})$ and its dynamics enter into the description through the term $\frac{\partial f}{\partial t}$; see Eq. (13). This difference between the EnKF and the present approach generates a quantitative difference.

The modelling of random processes in wildland fire propagation is embodied by the PDF $f(\boldsymbol{x} ; t \mid \overline{\boldsymbol{x}})$, accounting for the two independent random variables $(\overline{\boldsymbol{x}}+\chi)$ and $\xi$, which represent turbulence and fire spotting, respectively. The PDF $f$ is thus, in general, the convolution of the PDF associated with $(\overline{\boldsymbol{x}}+\chi)$, hereinafter labelled $G$, and the one associated with $\xi$, hereinafter labelled $q$. Some remarks are in order:

- To simplify the study of the present proof of concept, fire spotting is assumed to be independent of turbulence and to be a downwind phenomenon, even if these assumptions may not hold true in all cases and then not be entirely realistic.

- Embers are carried by the atmospheric mean wind $\boldsymbol{U}$ and they land at a certain distance $\ell$ from the fireline along the mean wind direction $\hat{\boldsymbol{n}}_{U}$. Hence, the effect of the randomly generated noise (hereafter referred to simply as noise) on model fire spotting $\xi$ is always aligned with the mean wind direction $\hat{\boldsymbol{n}}_{U}$, i.e. $\xi^{\omega}=\ell^{\omega} \hat{\boldsymbol{n}}_{U}$. Moreover, turbulent noise $\chi$ is a zero-mean noise, i.e. $\langle\chi\rangle=0$, while the fire spotting noise $\xi$ has a positive mean value, i.e. $\langle\ell\rangle>0$, the mean wind velocity $\boldsymbol{U}$ being the same in all realisations. Finally, the average position in the leeward sector is $\left\langle\boldsymbol{X}\left(t, \overline{\boldsymbol{x}}_{0}\right)\right\rangle=\overline{\boldsymbol{x}}=\overline{\boldsymbol{x}}_{\mathrm{ROS}}+\langle\ell\rangle \hat{\boldsymbol{n}}_{U}$, while in the windward sector it is $\left\langle\boldsymbol{X}\left(t, \overline{\boldsymbol{x}}_{0}\right)\right\rangle=\overline{\boldsymbol{x}}=\overline{\boldsymbol{x}}_{\mathrm{ROS}}$.

- It is also observed that since fire spotting is assumed to be a downwind phenomenon, the effect of fire spotting has to be taken into account only in the leeward part of the fireline:

$$
\begin{aligned}
& f(\boldsymbol{x} ; t \mid \overline{\boldsymbol{x}}) \\
& = \begin{cases}\int_{0}^{\infty} G\left(\boldsymbol{x}-\overline{\boldsymbol{x}}-\ell \hat{\boldsymbol{n}}_{U} ; t\right) q(\ell ; t) d \ell, & \text { if } \hat{\boldsymbol{n}} \cdot \hat{\boldsymbol{n}}_{U} \geq 0 \\
G(\boldsymbol{x}-\overline{\boldsymbol{x}} ; t), & \text { otherwise }\end{cases}
\end{aligned}
$$

The turbulent diffusion model can be derived by considering the scalar conservation equation. The model is determined by assuming a parameterisation of the turbulent heat fluxes. The most simple model is the Gaussian one that, in the isotropic case, is

$$
G(x-\bar{x} ; t)=\frac{1}{2 \pi \sigma^{2}(t)} \exp \left\{-\frac{(x-\bar{x})^{2}+(y-\bar{y})^{2}}{2 \sigma^{2}(t)}\right\},
$$


where $\boldsymbol{x} \equiv(x, y), \overline{\boldsymbol{x}} \equiv(\bar{x}, \bar{y})$, and $\sigma^{2}(t)=\left\langle(\boldsymbol{x}-\overline{\boldsymbol{x}})^{2}\right\rangle / 2$ is the particle displacement variance which is related to the turbulent diffusion coefficient $\mathcal{D}_{\mathrm{T}}$ by the law $\sigma^{2}(t)=2 \mathcal{D}_{\mathrm{T}} t$. In the present model, which is oversimplified because it is intended to investigate the potentiality of the proposed approach, the whole effect from turbulent processes with different scales, i.e. from the atmospheric boundary layer to the fire-induced flow, is assumed to be parameterised by the turbulent diffusion coefficient $\mathcal{D}_{\mathrm{T}}$ only.

The determination of the PDF of the downwind distribution of firebrands has been studied by numerical solution of balance equations (Sardoy et al., 2008; Kortas et al., 2009). Sardoy et al. (2008) found that the phenomenon follows a bimodal distribution, but only the firebrands with shortdistance landing were considered important for the analysis of danger related to fire spotting, since they have the potential to ignite a new fire, while those with a long-distance landing reach the ground in a charred oxidation state. Hence, longdistance landing distribution is neglected here. Furthermore, the frequency of the landing distance significantly increases with the separation from the source and, after a maximum value, gently decreases towards a minimum. In particular, it has been argued (Sardoy et al., 2008) that it follows a lognormal distribution

$q(\ell ; t)=\frac{1}{\sqrt{2 \pi} s(t) \ell} \exp \left\{-\frac{(\ln \ell-\mu(t))^{2}}{2 s(t)^{2}}\right\}$,

where $\mu(t)=\langle\ln \ell\rangle$ and $s(t)=\left\langle(\ln \ell-\mu(t))^{2}\right\rangle$ are, respectively, the mean and the standard deviation of $\ln \ell$. Another possible choice for $q$ (Kortas et al., 2009) is the Weibull distribution:

$q(\ell ; t)=\frac{h}{\lambda(t)}\left(\frac{\ell}{\lambda(t)}\right)^{h-1} \exp \left\{-\left(\frac{\ell}{\lambda(t)}\right)^{h}\right\}$,

where $h$, which depends on the firebrand shape, is established by experimental validation, and the mean value $\langle\ell\rangle$ is determined as $\langle\ell\rangle=\int_{0}^{\infty} \ell q(\ell ; t) d \ell=\lambda \Gamma(1+1 / h)$. When $h=2$, the Weibull distribution becomes the Rayleigh distribution that has been used for theoretical modelling (Wang, 2011).

The effects of turbulence on the present wildland fire propagation approach have been discussed previously (Pagnini and Massidda, 2012a, b). If a balanced Gaussian distribution is assumed and only turbulence is considered, the mean fireline position $\left\langle\boldsymbol{X}\left(t, \overline{\boldsymbol{x}}_{0}\right)\right\rangle$ is established according to the ROS $\mathcal{V}_{\text {ROS }}(\boldsymbol{x}, t)$, i.e. $\left\langle\boldsymbol{X}\left(t, \overline{\boldsymbol{x}}_{0}\right)\right\rangle=\overline{\boldsymbol{x}}(t)=\overline{\boldsymbol{x}}_{\mathrm{ROS}}(t)$ because $\langle\chi\rangle=0$. For a plane front $(\kappa=0)$, when the heating-before-burning mechanism is not taken into account and the threshold value $\varphi_{\mathrm{e}}^{\mathrm{th}}=0.5$ is assumed, it has been noted that the burned area $\Omega_{\mathrm{e}}$ grows more slowly than that determined by the levelset method (Pagnini and Massidda, 2012b). Instead, when pre-heating is considered, the advancement of the front is faster (Pagnini and Massidda, 2012b). Moreover, by taking turbulence into account, the fire flank and backing fire phenomena are also modelled (Pagnini and Massidda, 2012b).

When the contribution by fire spotting is taken into account, i.e. in the leeward fireline sector, it is easily seen that the advancement of the fireline is enhanced,

$$
\begin{aligned}
\left\langle\boldsymbol{X}\left(t, \overline{\boldsymbol{x}}_{0}\right)\right\rangle=\overline{\boldsymbol{x}}(t) & =\overline{\boldsymbol{x}}_{\mathrm{ROS}}(t)+\langle\chi\rangle+\langle\xi\rangle \\
& =\overline{\boldsymbol{x}}_{\mathrm{ROS}}(t)+\langle\ell(t)\rangle \hat{\boldsymbol{n}}_{U}
\end{aligned}
$$

since $\langle\ell(t)\rangle>0$ and $\hat{\boldsymbol{n}}_{U}$ is a unit vector pointing out of the burned domain, i.e. $\hat{\boldsymbol{n}} \cdot \hat{\boldsymbol{n}}_{U}>0$. As a consequence, when the fire spotting is included, the magnitude of the velocity of the mean fireline progression in the leeward sector is higher than the ROS, i.e.

$$
\begin{aligned}
\boldsymbol{V}(\overline{\boldsymbol{x}}, t)=\frac{\mathrm{d} \overline{\boldsymbol{x}}}{\mathrm{d} t} & =\frac{\mathrm{d}}{\mathrm{d} t}\left(\overline{\boldsymbol{x}}_{\mathrm{ROS}}(t)+\langle\ell\rangle \hat{\boldsymbol{n}}_{U}\right) \\
& =\mathcal{V}_{\mathrm{ROS}}(\overline{\boldsymbol{x}} t) \hat{\boldsymbol{n}}+\frac{\mathrm{d}\langle\ell\rangle}{\mathrm{d} t} \hat{\boldsymbol{n}}_{U}+\langle\ell\rangle \frac{\mathrm{d} \hat{\boldsymbol{n}}_{U}}{\mathrm{~d} t} \\
& =\boldsymbol{V}_{\mathrm{ROS}}(\overline{\boldsymbol{x}}, t)+\boldsymbol{V}_{\ell}(\overline{\boldsymbol{x}}, t) .
\end{aligned}
$$

The above result, expressed by Eq. (29), is a key feature of the proposed approach because it determines the correction $\boldsymbol{V}_{\ell}(\overline{\boldsymbol{x}}, t)$ due to the fire spotting phenomenon that affects the fireline velocity. The latter, in fact, is generally assumed to include only the ROS contribution, i.e. $\boldsymbol{V}_{\operatorname{ROS}}(\overline{\boldsymbol{x}}, t)=\mathcal{V}_{\mathrm{ROS}}(\boldsymbol{x}, t) \hat{\boldsymbol{n}}$. It is remarked here that the new additional terms appearing in Eq. (29) are independent of the procedure for the determination of the ROS, and the level-set equation for the leeward sector turns out to be

$$
\frac{\partial \gamma}{\partial t}=\left(\mathcal{V}_{\mathrm{ROS}}+\boldsymbol{V}_{\ell} \cdot \hat{\boldsymbol{n}}\right)|\nabla \gamma| \text {. }
$$

Another important result of the proposed approach is the possibility of managing real-world cases in which fire overcomes a zone without fuel, like roads, fire-break lines, and rivers. This valuable feature of the model has also been observed in the case in which only turbulence was taken into account (Pagnini and Massidda, 2012a, b). In the classical level-set method, this issue cannot be solved, because when there is no fuel the velocity field is null too, i.e. $\boldsymbol{V}(\boldsymbol{x}, t)=0$, and the fire front stops. Indeed, when the fuel is null, the fireline spreading is driven by the action of the turbulent motion of the hot air and, in the leeward sector of the fireline, also by the presence of embers carried by the wind. Hence, the fire propagates according to the following diffusion-type equation following from Eq. (16) by setting $\boldsymbol{V}(\boldsymbol{x}, t)=0$, i.e.

$$
\frac{\partial \varphi_{\mathrm{e}}}{\partial t}=\mathcal{E} \varphi_{\mathrm{e}}
$$

\section{Numerical results}

The modelling approach discussed qualitatively in the previous section is now analysed by means of numerical simulations. For this purpose, a C/OpenMP code has been developed starting from a $\mathrm{C}$ code previously developed and 
Table 1. Values of the parameters of the model which are kept fixed throughout the numerical simulation discussed here.

\begin{tabular}{ll}
\hline Fixed simulation parameters & Value \\
\hline Fuel low heat of combustion, $H$ & $22000 \mathrm{~kJ} \mathrm{~kg}^{-1}$ \\
Oven-dry mass of fuel, $\omega_{0}$ & $2.243 \mathrm{~kg} \mathrm{~m}^{-2}$ \\
Ambient gas density, $\rho_{\infty}$ & $1.1 \mathrm{~kg} \mathrm{~m}^{-3}$ \\
Ambient gas temperature, $T_{\mathrm{a}}$ & $300 \mathrm{~K}$ \\
Mean specific heat of gas, $c_{\mathrm{pg}}$ & $1121 \mathrm{~kJ}(\mathrm{~kg} \mathrm{~K})^{-1}$ \\
Gravitational acceleration, $g$ & $9.81 \mathrm{~m} \mathrm{~s}^{-2}$ \\
Tree torching intensity, $I_{\mathrm{t}}$ & $0.015 \mathrm{~kW} \mathrm{~m}^{-1}$ \\
Turbulent diffusion coefficient, $\mathcal{D}_{\mathrm{T}}$ & $0.04 \mathrm{~m}^{2} \mathrm{~s}^{-1}$ \\
Ignition delay of hot air, $\tau_{\mathrm{h}}$ & $600 \mathrm{~s}$ \\
Ignition delay of firebrands, $\tau_{\mathrm{f}}$ & $60 \mathrm{~s}$ \\
Width of the fire break in the windward sector & $60 \mathrm{~m}$ \\
Width of the fire break in the leeward sector & $90 \mathrm{~m}$ \\
\hline
\end{tabular}

successfully employed for the analysis of the turbulence effects by Pagnini and Massidda (2012b, a). The present code, still under active development and to be described thoroughly elsewhere in the future, aims at being a general-purpose code allowing for the simulation of wildfire propagation under a large variety of atmospheric and environmental conditions, including realistic fire breaks and coupled atmosphere-fire flow fields of practical interest.

Since the aim of the present paper is a proof of concept to demonstrate the potential of the present approach, rather than to simulate wildland fire behaviour under realistic conditions, the numerical results presented in the following are restricted to oversimplified cases chosen to highlight the main features of the model. For this purpose, the results obtained with the full-feature model are compared to those obtained in the absence of the fire spotting effects, as well as to those obtained by adopting the classical approach involving a deterministic front propagation (i.e. with the classical level-set method). Moreover, the test cases are also chosen in a way so as to facilitate the comparison with results available in the literature, obtained by means of different approaches.

\subsection{Simulation set-up}

A fireline propagating on a flat terrain covered by an idealised Pinus ponderosa ecosystem has been selected for simulation, following previous analyses by Sardoy et al. (2007, 2008) and Perryman et al. (2013) of the same issues.

The initial fireline $\Gamma_{0}$ is assumed to be circular, and the maximum value of the ROS, $\mathcal{V}_{0}$, is estimated by means of the Byram formula (Byram, 1959; Alexander, 1982):

$\mathcal{V}_{0}=\frac{I}{H \omega_{0}}$,

where $I$ is the surface fireline intensity, $H$ is the fuel low heat of combustion and $\omega_{0}$ is the oven-dry mass of fuel consumed per unit area in the active flaming zone (all the numerical values are given in Tables 1 and 2).
Table 2. Values of the mean wind velocity, $U_{\mathrm{t}}$, of the fire intensity, $I$, and of the $F r$ number for the four cases for which numerical results are presented here. These values correspond to the same system configurations considered by Sardoy et al. (2008).

\begin{tabular}{lrcc}
\hline Case & $U_{\mathrm{t}}\left[\mathrm{m} \mathrm{s}^{-1}\right]$ & $I\left[\mathrm{~kW} \mathrm{~m}^{-1}\right]$ & $F r[-]$ \\
\hline $\mathcal{A}$ & 6.7 & 10000 & 10.4 \\
$\mathcal{B}$ & 6.7 & 30000 & 7.2 \\
$\mathcal{C}$ & 17.88 & 10000 & 27.8 \\
$\mathcal{D}$ & 17.88 & 30000 & 19.3 \\
\hline
\end{tabular}

The functional dependence of the ROS on the wind is taken into account through a corrective factor $f_{\mathrm{W}}$ as follows:

$\mathcal{V}(\boldsymbol{x}, t)=\mathcal{V}_{0} \frac{\left(1+f_{\mathrm{W}}\right)}{\alpha}$,

where $f_{\mathrm{W}}$ is computed following the prescription of the fireLib and Fire Behaviour SDK libraries (http://fire.org; see also Mandel et al., 2011), in the case of the NFFL (Northern Forest Fire Laboratory) Model 9, and $\alpha$ is a suitable parameter for guaranteeing that the maximum ROS equals the ROS prescribed by the Byram formula (Eq. 32).

The mean wind is assumed to be constant both in direction, $\hat{\boldsymbol{n}}$, and velocity, $U_{\mathrm{t}}$, in order to highlight the effects of the fire spotting. In particular, in all the plotted results the wind is directed along the positive $x$ direction (i.e. $\hat{\boldsymbol{n}} \equiv \hat{\boldsymbol{i}}$ ), and the wind velocity, $U_{\mathrm{t}}$, is intended to be the velocity measured at the top of the tree canopy that is assumed to be $10 \mathrm{~m}$ high, as by Sardoy et al. (2008).

The turbulent heat transfer is modelled by means of the Gaussian distribution, see Eq. (25), and firebrand landing is modelled, following Sardoy et al. (2007, 2008), by means of a log-normal distribution, as given in Eq. (26). In this simplified analysis, the turbulent diffusion coefficient $\mathcal{D}_{\mathrm{T}}$ and ignition delays of the hot air and the firebrands are assumed to be constant throughout the numerical simulations. In particular, it is well known that the value of thermal diffusivity in air is around $2 \times 10^{-5} \mathrm{~m}^{2} \mathrm{~s}^{-1}$; the effect of turbulence is then accounted for here by generating a turbulent diffusion coefficient of three orders of magnitude higher, i.e. $\mathcal{D}_{\mathrm{T}}=4 \times 10^{-2} \mathrm{~m}^{2} \mathrm{~s}^{-1}$. This value has also been chosen in view of the analysis of the role and effects of firebrands. A more detailed study of turbulence effects with higher values of $\mathcal{D}_{\mathrm{T}}$ has been performed by Pagnini and Massidda (2012b, a). Moreover, in the following simulations, the potentiality of the proposed approach has been studied for simplicity, with the assumption that the ignition delay associated with firebrands is much smaller than that associated with hot air; hence, $\tau_{\mathrm{h}} \gg \tau_{\mathrm{f}}$ and $\tau \simeq \tau_{\mathrm{f}}$ holds. All the chosen values are given in Table 1. 

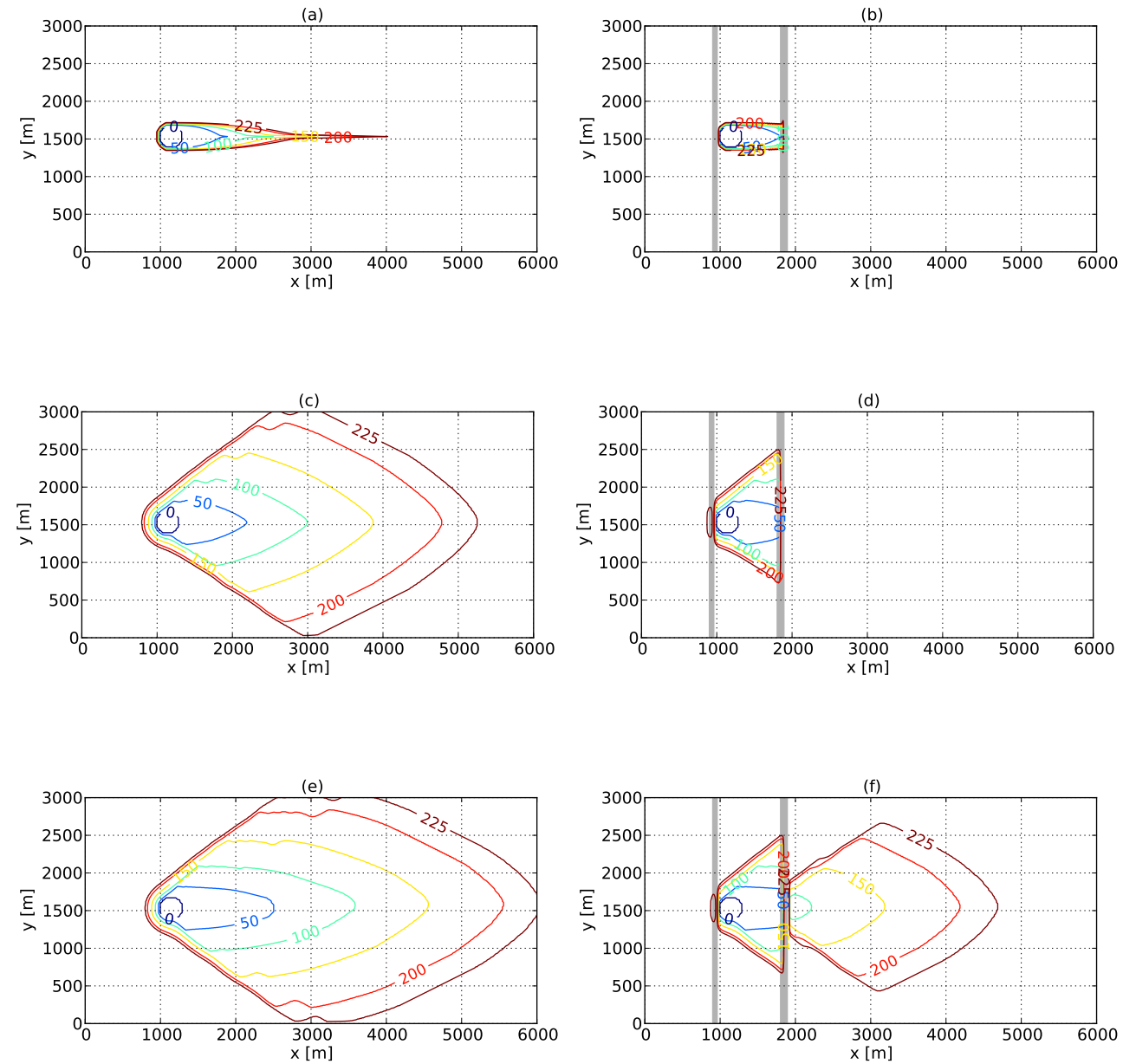

Figure 1. Time evolution of the firefront when the mean wind velocity and the fire intensity are $U_{\mathrm{t}}=6.70 \mathrm{~m} \mathrm{~s}^{-1}$ and $I=10000 \mathrm{~kW} \mathrm{~m}^{-1}$ (case $\mathcal{A}$ ), in the absence (on the left) and the presence (on the right) of two fire-break zones (grey stripes) located on the left and on the right of the initial firefront. The results are obtained by adopting the level-set method (top row panels) by the present modelling approach when only turbulence is taken into account (middle row panels), and when both turbulence and fire spotting are considered (bottom row panels). The labels on the contour lines represent the propagation time (expressed in minutes). All the parameters of the model are given in Table 1.

Concerning fire spotting modelling, Sardoy et al. (2008) distinguish two landing regimes according to the Froude number $F r=U_{\mathrm{t}} / \sqrt{g L_{\mathrm{c}}}$, where $g$ is the gravitational acceleration and $L_{\mathrm{c}}$ is the characteristic length of the plume convecting embers, calculated by $L_{\mathrm{c}}=\left(I /\left(\rho_{\infty} c_{\mathrm{pg}} T_{\mathrm{a}} \sqrt{g}\right)\right)^{2 / 3}$, where $\rho_{\infty}, T_{\mathrm{a}}$ and $c_{\mathrm{pg}}$ are, respectively, the ambient gas density and temperature and the specific heat of the gas. The two mentioned regimes are the buoyancy-driven regime $(F r<1)$ and the wind-driven regime $(F r>1)$. In particular, following the fitting of numerical data generated by Sardoy et al. (2008) when the char content is $v_{\mathrm{c}}=0.39$, Perryman et al. (2013) suggest the following pairs of parameters:

- buoyancy-driven regime $(F r<1)$

$$
\begin{aligned}
& \mu=1.47 I_{\mathrm{f}}^{0.54} U_{\mathrm{t}}^{-0.55}+1.14, \\
& s=0.86 I_{\mathrm{f}}^{-0.21} U_{\mathrm{t}}^{0.44}+0.19,
\end{aligned}
$$

- wind-driven regime $(F r>1)$

$$
\begin{aligned}
& \mu=1.32 I_{\mathrm{f}}^{0.26} U_{\mathrm{t}}^{0.11}-0.02, \\
& s=4.95 I_{\mathrm{f}}^{-0.01} U_{\mathrm{t}}^{-0.02}-3.48,
\end{aligned}
$$

where $U_{\mathrm{t}}$ must be given in $\mathrm{m} \mathrm{s}^{-1}$, and $I_{\mathrm{f}}$, given in $\mathrm{kW} \mathrm{m}^{-1}$, represents the fire intensity enriched by the tree torching intensity $I_{\mathrm{t}}$, i.e. $I_{\mathrm{f}}=I+I_{\mathrm{t}}$.

It is well known that, in the log-normal density, the increasing of the value of the mean $\mu$ corresponds to a slower decay of the right tail, i.e. for $\ell \rightarrow \infty$, and correspondingly a faster decay for the left tail, i.e. $\ell \rightarrow 0$, which means a higher probability of having a large value of $\ell$. Indeed, an increase in the value of the standard deviation $s$ corresponds to a left shift of the maximum value of the probability density, which means that the most frequent event has a small value of $\ell$. 

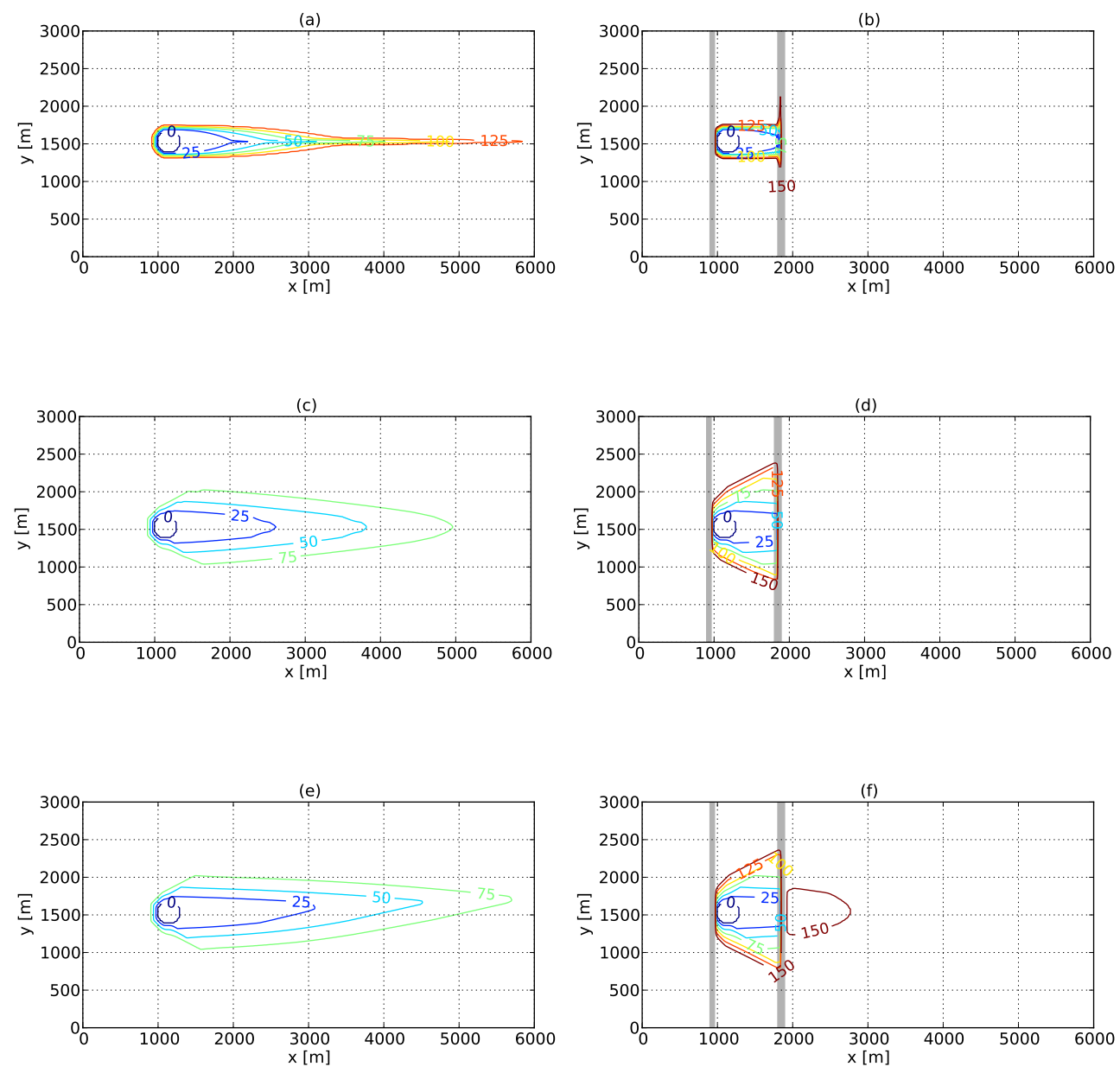

Figure 2. The same as in Fig. 1, but when the mean wind velocity and the fire intensity are $U_{\mathrm{t}}=6.70 \mathrm{~m} \mathrm{~s}^{-1}$ and $I=30000 \mathrm{~kW} \mathrm{~m}^{-1}($ case $\mathcal{B})$.

As previously pointed out, simulations are performed following some of the case studies considered by other authors, in particular by Sardoy et al. (2008). With the purpose of analysing the main features of the model proposed here, four cases have been regarded as worthy of discussion; these four cases correspond to the possible combinations of two selected values of the fire intensity $I$ and two selected values of the mean wind velocity $U_{\mathrm{t}}\left(I=10000-30000 \mathrm{~kW} \mathrm{~m}^{-1}\right.$; $U_{\mathrm{t}}=6.7-17.88 \mathrm{~m} \mathrm{~s}^{-1}$ ). In Table 2 , these four cases (named cases $\mathcal{A}, \mathcal{B}, \mathcal{C}$ and $\mathcal{D}$ ) are defined properly. It should be noted that, despite the fact that a wind velocity of $17.88 \mathrm{~m} \mathrm{~s}^{-1}$ may appear very high, this value has been chosen so as to favour the comparison with results published by other authors (Sardoy et al., 2008).

As mentioned earlier, in all the four cases under investigation, numerical simulations have been performed assuming a deterministic front propagation, i.e. neglecting turbulence and the fire spotting phenomenon, and assuming a random front propagating both in the presence and absence of the fire spotting phenomenon.
Moreover, in all cases, simulations have been carried out assuming that the wildland fire freely propagates on the flat terrain, as well as introducing two fire breaks, the latter being modelled as two combustible-free stripes of terrain perpendicular to the wind direction located windward and leeward with respect to the initial fire location.

As a result, for each of the four test cases, the results of a set of six numerical simulations are presented and collectively discussed.

\subsection{Discussion}

The results of the numerical simulations corresponding to the four cases introduced and summarised previously in Table 2 are shown in Figs. 1, 2, 3 and 4, respectively.

In each of the figures, the evolutions of the fireline freely propagating in a terrain with no fire breaks (i.e. fuel-free regions) are shown on the left, and the corresponding evolution in the presence of two fire breaks is shown on the right, being the fire breaks represented by grey vertical stripes of different widths (see Table 1 for the values of all the model parameters), i.e. perpendicular to the wind direction. For both cases 

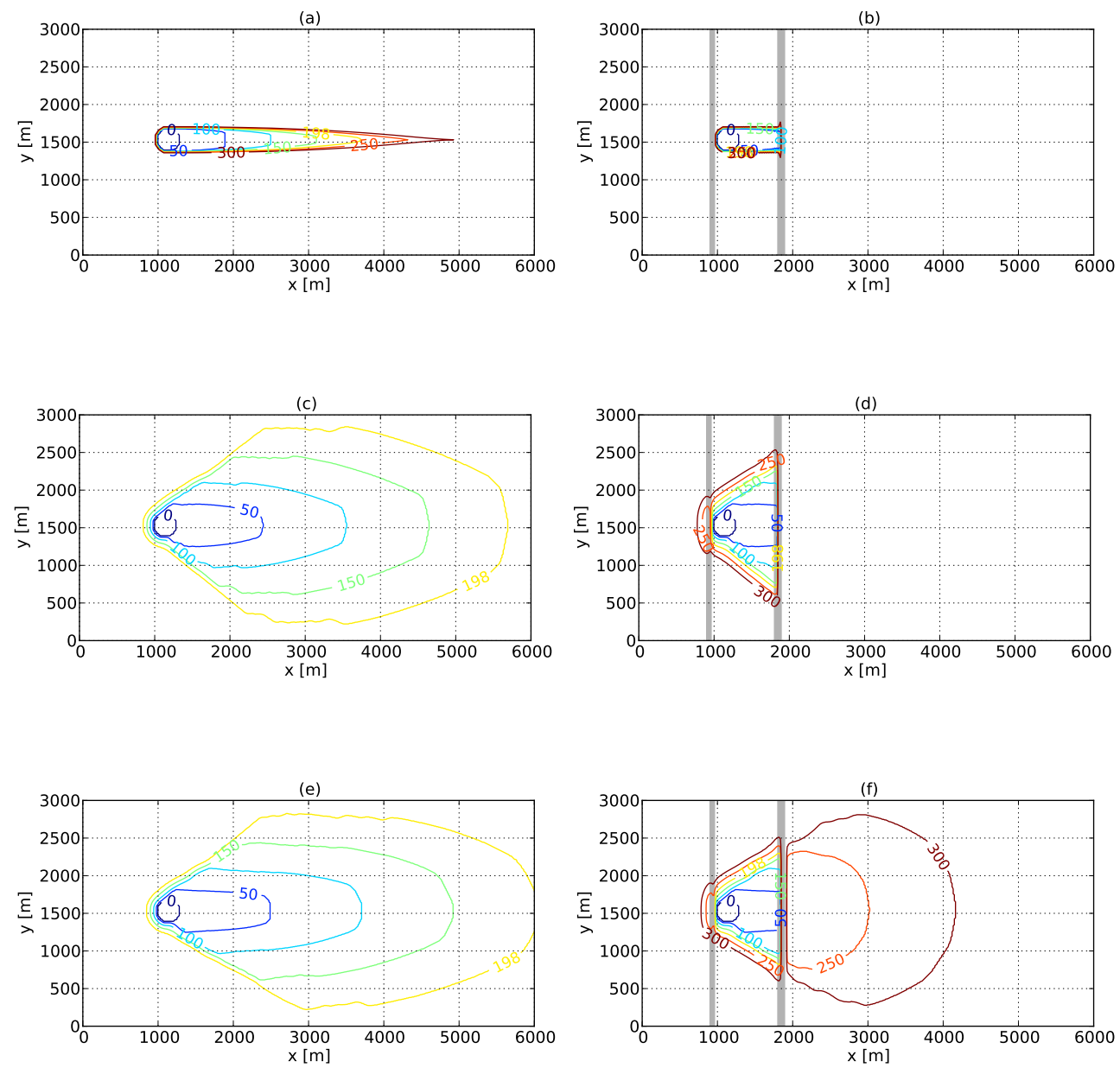

Figure 3. The same as in Fig. 1, but when the mean wind velocity and the fire intensity are $U_{\mathrm{t}}=17.88 \mathrm{~m} \mathrm{~s}^{-1}$ and $I=10000 \mathrm{~kW} \mathrm{~m}^{-1}($ case $\mathcal{C}$ ).

(without and with fire breaks), the results obtained by adopting three different models are shown in the figures: the deterministic model, in which the firefront is tracked by means of the classical level-set method (top row of each figure); the model in which the front is tracked by means of the randomised level-set method, including only the turbulence effects (middle row); and the full-featured model presented in the previous section, in which the fire spotting phenomenon is also included (bottom row).

In general, it is possible to note the high number, the variability and the complexity of phenomenological situations that the present approach can handle, as well as the strong sensitivity to different framework features.

As a general rule, by comparison of the results obtained in the randomised approach to those obtained in the deterministic framework, it is possible to state that, as expected, the firefront propagates faster when turbulence effects are taken into account. Moreover, when fire spotting effects are also included in the model, the firefront propagates even faster, compared with results obtained with the model that include only the turbulence effects. These four cases, displayed in
Figs. 1, 2, 3 and 4, show that the differences between cases are the consequences of the air pre-heating action due to the heat transfer mechanism enhanced by turbulence, and of the rapid ignition connected to embers landing in the yet-to-burn region ahead of the fireline front.

Moreover, fire flanking and backing fire appear well simulated.

Even though it should be remarked that the purpose of this analysis is limited to a first-look investigation of the capabilities of the model, and no attempt has been made in order to choose the model parameters in a realistic way, the effects of the fire spotting phenomenon still appear relevant and worthy of being taken into account in any model aiming at a realistic simulation of the behaviour of wildland fire.

The presented numerical results, in fact, strongly support the importance of the fire spotting phenomenon as a mechanism enhancing the frontline propagation. This is particularly evident in the cases in which the fire propagates in a region in which fire breaks are present. In this situation, the modelling results strikingly point out how the fire spotting phenomenon may be crucial in making the fire overcome the fire breaks 

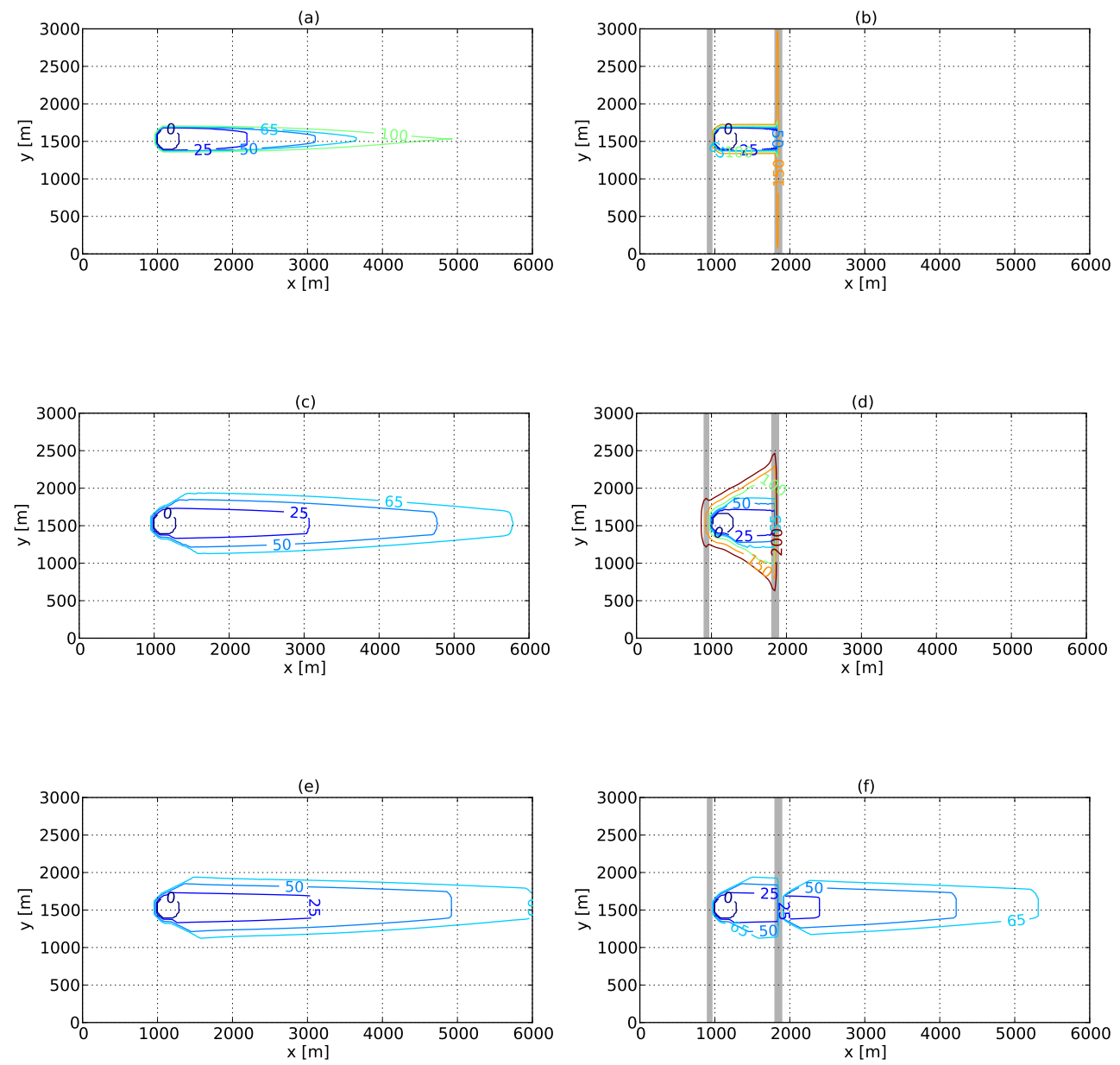

Figure 4. The same as in Fig. 1, but when the mean wind velocity and the fire intensity are $U_{\mathrm{t}}=17.88 \mathrm{~m} \mathrm{~s}^{-1}$ and $I=30000 \mathrm{~kW} \mathrm{~m}^{-1}(\mathrm{case} \mathcal{D}$ ).

faster than when adopting a model that includes only turbulence effects. As has been shown previously (Pagnini and Massidda, 2012b, a), the turbulence itself can be responsible for the spreading of the wildland fire across fire breaks, but it appears clearly, when comparing the results of Figs. 1d, 2d, $3 \mathrm{~d}$ and $4 \mathrm{~d}$ to the corresponding ones of Figs. 1f, $2 \mathrm{f}, 3 \mathrm{f}$ and $4 \mathrm{f}$, that the fire spotting phenomenon is capable of enhancing remarkably this capability of the wildland fire. It is worth noting here that, since the present analysis is primarily devoted to the investigation of the main feature of the new model, including fire spotting effects, the numerical results are presented for short-time propagation of the fire, in contrast to the results discussed in Pagnini and Massidda (2012b), in which, being the focus of the analysis of the turbulence effects, the numerical results concerned long-term propagation.

\section{Conclusions}

An approach to tracking random fronts (Pagnini and Bonomi, 2011; Pagnini and Massidda, 2012b, a) has been described, re-arranged and analysed to study its suitability for investigating the effects of random processes on wildland fire propagation. Actually, the random fireline is modelled in terms of an average position determined by a level-set model with a certain ROS, and the statistical spread is determined by the PDF of displacements of random contour points marked as active flame holders.

This formulation is similar to the so-called SPH theory (Monaghan, 2005), where a kernel function with a smoothing length is introduced to study non-smooth solutions. In the present approach, non-smooth solutions obtained by the level-set equation are weighted by a kernel function with a smoothing length that straightforwardly follows to be determined by the PDF of contour points.

This approach is a generalisation of the level-set method that permits the tracking of even random fronts, and the effective fireline contours emerge to be governed by a 
reaction-diffusion-type equation. This last fact reconciles the two widely used approaches to studying wildland fire propagation, namely the one based on the level-set method with a given ROS, and the one based on reaction-diffusion equations.

In previous analyses (Pagnini and Massidda, 2012b, a), only turbulence effects were considered. Here, random effects due to the fire spotting phenomenon have also been taken into account. In this respect, in order to consider the statistical effect due to fire spotting, a formula is stated to modify the velocity of the average frontline driven by the level-set equation.

Numerical simulations of a simple case study are performed to explore the model behaviour. Fire spotting parameterisation follows numerical results by Sardoy et al. (2008) combined with arguments by Perryman et al. (2013), and the maximum value of the ROS has been estimated by the Byram formula (Byram, 1959; Alexander, 1982). The values for the turbulent diffusion coefficient and ignition delay have been opportunely chosen to highlight the role of each single phenomenon and the structure of their joint action better.

In particular, for the same given ROS, the model shows a faster fireline propagation with respect to the level-set formulation and, in opposition to the level-set-based modelling, the randomisation permits the modelling of backing fire, fire flanking and fire that overcome an obstacle without fuel.

Moreover, since ignition delay for fire spotting is stated as being shorter than for heating, a further increasing of the propagation speed is generated in the direction of ember landings, which are assumed to be downwind.

The role of the fire intensity and the mean wind were also analysed. The effect due to the increasing of the fire intensity emerges as being stronger than that due to the increasing of the mean wind to propagate the fire faster. This is a direct consequence of the ROS estimation.

To conclude, this formulation emerges as being more suitable than the ordinary level-set approach for managing realworld dangerous situations related to the random character of wildland fire propagation. In fact, this modelling approach allows for the prediction of fire flanking, backing fire, and faster fire propagation as a consequence of the preheating action by the hot air and the firebrand landing, and has the paramount property of reproducing the overcoming of a break fire without fuel by the fire because of the diffusion of the hot air (Pagnini and Massidda, 2012b, a) and ember jumping. The validation of the present modelling approach with realistic parameters of turbulence and ignition delay will be the topic of further future research.

Acknowledgements. This research was developed in the framework of the GNFM/INdAM Young Researchers Project 2013 Hyperbolic modelling into the extended thermodynamics framework to study combustion-wave interactions in turbulent premixed combustion and it has also supported by Bizkaia Talent Grant AYD-000-226 Combustion-wave interactions via extended thermodynamics co-funded by Bizkaia Xede and European Commission (CO-FUND programme 2014/2016), by the Basque Government through the BERC 2014-2017 program and by the Spanish Ministry of Economy and Competitiveness MINECO: BCAM Severo Ochoa accreditation SEV-2013-0323.

Edited by: D. Veynante

Reviewed by: two anonymous referees

\section{References}

Alexander, M. E.: Calculating and interpreting forest fire intensities, Can. J. Bot., 60, 349-357, 1982.

Almeida, R. M. and Macau, E. E. N.: Stochastic cellular automata model for wildland fire spread dynamics, J. Phys. Conf. Ser., 285, 012038, doi:10.1088/1742-6596/285/1/012038, 2011.

Asensio, M. I. and Ferragut, L.: On a wildland fire model with radiation, Int. J. Numer. Meth. Engng., 54, 137-157, 2002.

Babak, P., Bourlioux, A., and Hillen, T.: The effect of wind on the propagation of an idealized forest fire, SIAM J. Appl. Math., 70, 1364-1388, 2009.

Balbi, J. H., Rossi, J. L., Marcelli, T., and Santoni, P. A.: A 3D physical real-time model of surface fires across fuel beds, Combust. Sci. Technol., 179, 2511-2537, 2007.

Balbi, J. H., Morandini, F., Silvani, X., Filippi, J. B., and Rinieri, F.: A physical model for wildland fires, Combust. Flame, 156, 2217-2230, 2009.

Beezley, J. D. and Mandel, J.: Morphing ensemble Kalman filters, Tellus A, 60, 131-140, 2008.

Beezley, J. D., Chakraborty, S., Coen, J. L., Douglas, C. C., Mandel, J., Vodacek, A., and Wang, Z.: Real-Time Data Driven Wildland Fire Modeling, in: Computational Science, ICCS 2008, 8th International Conference, Kraków, Poland, 23-25 June 2008, Proceedings, Part III, edited by: Bubak, M., van Albada, G. D., Dongarra, J., and Sloot, P. M. A., vol. 5103 of Lecture Notes in Computer Science, Springer-Verlag, Berlin, Heidelberg, 46-53, 2008.

Bhutia, S., Jenkins, M. A., and Sun, R.: Comparison of firebrand propagation prediction by a plume model and a coupled fire/atmosphere large-eddy simulator, J. Adv. Model. Earth Syst., 2, 4, doi:10.3894/JAMES.2010.2.4, 2010.

Boychuk, D., Braun, W. J., Kulperger, R. J., Krougly, Z. L., and Stanford, D. A.: A stochastic forest fire growth model, Environ. Ecol. Stat., 16, 133-151, 2009.

Byram, G. M.: Combustion of Forest Fuels, in: Forest Fire: Control and Use, edited by: Davis, K. P., McGraw Hill, New York, 61-89, 1959.

Clark, T. L., Jenkins, M. A., Coen, J., and Packham, D.: A coupled atmospheric-fire model: convective feedback on fire-line dynamics, J. Appl. Meteorol., 35, 875-901, 1996.

Clements, C. B., Zhong, S., Bian, X., Heilman, W. E., and Byun, D. W.: First observations of turbulence generated by grass fires, J. Geophys. Res., 113, D22102, doi:10.1029/2008JD010014, 2008.

Cobb, J. M. L. and Beezley, J. D.: On the convergence of the ensemble Kalman filter, Appl. Math., 56, 533-541, 2011.

Coen, J. J., Cameron, M., Michalakes, J., Patton, E. G., Riggan, P. J., and Yedinak, K. M.: WRF-Fire: coupled weather-wildland fire modeling with the Weather Research and Forecasting model, J. Appl. Meteorol. Clim., 52, 16-38, 2013. 
Cunningham, P. and Linn, R. R.: Numerical simulations of grass fires using a coupled atmosphere-fire model: Dynamics of fire spread, J. Geophys. Res., 112, D05108, doi:10.1029/2006JD007638, 2007.

Dobrinkova, N., Jordanov, G., and Mandel, J.: WRF-Fire Applied in Bulgaria, in: Numerical Methods and Applications, NMA 2010, 7th International Conference, Borovets, Bulgaria, 20-24 August 2010, edited by: Ivan Dimov, S. D. and Kolkovska, N., vol. 6046 of Lecture Notes in Computer Science, SpringerVerlag, Berlin, Heidelberg, 133-140, 2011.

Favier, C.: Percolation model of fire dynamic, Phys. Lett. A, 330, 396-401, 2004.

Filippi, J. B., Bosseur, F., Mari, C., Lac, C., Moigne, P. L., Cuenot, B., Veynante, D., Cariolle, D., and Balbi, J. H.: Coupled atmosphere-wildland fire modelling, J. Adv. Model. Earth Syst., 1, 11, doi:10.3894/JAMES.2009.1.11, 2009.

Filippi, J. B., Morandini, F., Balbi, J. H., and Hill, D.: Discrete event front tracking simulator of a physical fire spread model, Simulation, 86, 629-646, 2010.

Filippi, J. B., Bosseur, F., Pialat, X., Santoni, P. A., Strada, S., and Mari, C.: Simulation of coupled Fire/Atmosphere interaction with the MesoNH-ForeFire models, J. Combust., 2011, 540390, doi:10.1155/2011/540390, 2011.

Filippi, J. B., Pialat, X., and Clements, C. B.: Assessment of ForeFire/Meso-NH for wildland fire/atmosphere coupled simulation of the FireFlux experiment, Proc. Combust. Inst., 34, 26332640, 2013.

Finney, M.: Fire growth using minimum travel time methods, Can. J. For. Res., 32, 1420-1424, 2002.

Finney, M.: Calculation of fire spread rates across random landscapes, Int. J. Wildland Fire, 12, 167-174, 2003.

Forthofer, J. M. and Goodrick, S. L.: Review of vortices in wildland fire, J. Combust., 2011, 984363 doi:10.1155/2011/984363, 2011.

Hunt, H.: A new conceptual model for forest fires based on percolation theory, Complexity, 13, 12-17, 2007.

Klimontovich, Y. L.: Nonlinear Brownian motion, PhysicsUspekhi, 37, 737-767, 1994.

Koo, E., Pagni, P. J., Weise, D. R., and Woycheese, J. P.: Firebrands and spotting ignition in large-scale fires, Int. J. Wildland Fire, 19, 818-843, 2010.

Kortas, S., Mindykowski, P., Consalvi, J. L., Mhiri, H., and Porterie, B.: Experimental validation of a numerical model for the transport of firebrands, Fire Safety J., 44, 1095-1102, 2009.

Linn, R. R. and Cunningham, P.: Numerical simulations of grass fires using a coupled atmosphere-fire model: Basic fire behavior and dependence on wind speed, J. Geophys. Res., 110, D13107, doi:10.1029/2004JD005597, 2005.

Mallet, V., Keyes, D. E., and Fendell, F. E.: Modeling wildland fire propagation with level set methods, Comput. Math. Appl., 57, 1089-1101, 2009.

Mandel, J., Bennethum, L. S., Beezley, J. D., Coen, J. L., Douglas, C. C., Kim, M., and Vodacek, A.: A wildland fire model with data assimilation, Math. Comput. Simulat., 79, 584-606, 2008.

Mandel, J., Beezley, J. D., Coen, J. L., and Kim, M.: Data assimilation for wildland fires: Ensemble Kalman filters in coupled atmosphere-surface models, IEEE Control Syst. Mag., 29, 47$65,2009$.
Mandel, J., Beezley, J. D., and Kochanski, A. K.: Coupled atmosphere-wildland fire modeling with WRF 3.3 and SFIRE 2011, Geosci. Model Dev., 4, 591-610, doi:10.5194/gmd4-591-2011, 2011.

Monaghan, J. J.: Smoothed particle hydrodynamics, Rep. Prog. Phys., 68, 1703-1759, 2005.

Montenegro, R., Plaza, A., Ferragut, L., and Asensio, M. I.: Application of a nonlinear evolution model to fire propagation, Nonlin. Anal. Theory Meth. Appl., 30, 2873-2882, 1997.

Morgante, A. E.: Incorporating Spotting into a Simple Fire Perimeter Model, Master's thesis, Humboldt State University, Arcata, CA, 2011.

Pagnini, G. and Bonomi, E.: Lagrangian formulation of turbulent premixed combustion, Phys. Rev. Lett., 107, 044503, doi:10.1103/PhysRevLett.107.044503, 2011.

Pagnini, G. and Massidda, L.: The randomized level-set method to model turbulence effects in wildland fire propagation, in: Modelling Fire Behaviour and Risk, edited by: Spano, D., Bacciu, V., Salis, M., and Sirca, C., Proceedings of the International Conference on Fire Behaviour and Risk, ICFBR 2011, 4-6 October 2011, Alghero, Italy, 126-131, 2012a.

Pagnini, G. and Massidda, L.: Modelling turbulence effects in wildland fire propagation by the randomized level-set method, Technical Report 2012/PM12a, CRS4, Pula (CA), Sardinia, Italy, July 2012, revised version: August 2014, http://publications. crs4.it/pubdocs/2012/PM12a/pagnini_massidda-levelset.pdf and arXiv:1408.6129 (last access: 25 August 2014), 2012 b.

Perryman, H. A.: A Mathematical Model of Spot Fires and their Management Implications, Master's thesis, Humboldt State University, Arcata, CA, 2009.

Perryman, H. A., Dugaw, C. J., Varner, J. M., and Johnson, D. L.: A cellular automata model to link surface fires to firebrand lift-off and dispersal, Int. J. Wildland Fire, 22, 428-439, 2013.

Potter, B. E.: A dynamics based view of atmosphere-fire interactions, Int. J. Wildland Fire, 11, 247-255, 2002.

Potter, B. E.: Atmospheric interactions with wildland fire behaviour, I. Basic surface interactions, vertical profiles and synoptic structures, Int. J. Wildland Fire, 21, 779-801, 2012a.

Potter, B. E.: Atmospheric interactions with wildland fire behaviour, II. Plume and vortex dynamics, Int. J. Wildland Fire, 21, 802817, 2012b.

Rehm, R. G. and McDermott, R. J.: Fire-Front Propagation Using the Level Set Method, Tech. Note 1611, Natl. Inst. Stand. Technol., Gaithersburg, MD, USA, 2009.

Rochoux, M. C., Ricci, S., Lucor, D., Cuenot, B., Trouvé, A., and Bart, J.-M.: Towards predictive simulation of wildfire spread using a reduced-cost Ensemble Kalman Filter based on Polynomial Chaos approximation, in: Studying Turbulence Using Numerical Simulation Databases - XIV. Proceedings of the Summer Program 2012, edited by: Moin, P. and Nichols, J., Center for Turbulence Research, Stanford University, California, USA, 2012.

Rochoux, M. C., Emery, C., Ricci, S., Cuenot, B., and Trouvé, A.: Towards predictive simulations of wildfire spread at regional scale using ensamble-based data assimilation to correct the fire front position, in: Proceedings of the Eleventh International Symposium on Fire Safety Science, International Association for Fire Safety Science, 10-14 February 2014, University of Canterbury, New Zealan, 2013. 
Rochoux, M. C., Ricci, S., Lucor, D., Cuenot, B., and Trouvé, A.: Towards predictive data-driven simulations of wildfire spread - Part I: Reduced-cost Ensemble Kalman Filter based on a Polynomial Chaos surrogate model for parameter estimation, Nat. Hazards Earth Syst. Sci. Discuss., 2, 3289-3349, doi:10.5194/nhessd-2-3289-2014, 2014a.

Rochoux, M. C., Emery, C., Ricci, S., Cuenot, B., and Trouvé, A.: Towards predictive data-driven simulations of wildfire spread Part 2: Ensemble Kalman Filter for the state estimation of a fronttracking simulator of wildfire spread, Nat. Hazards Earth Syst. Sci. Discuss., 2, 3769-3820, doi:10.5194/nhessd-2-3769-2014, 2014b.

Rothermel, R. C.: A Mathematical Model for Predicting Fire Spread in Wildland Fires, Tech. Rep. Research Paper INT-115, USDA Forest Service, Intermountain Forest and Range Experiment Station, Ogden, Utah, available at: http://www.iafss. org/publications/fss/11/author/11125 and http://www.iafss.org/ publications/fss/11/217/view (last acces: 13 August 2014), 1972.

Sardoy, N., Consalvi, J. L., Porterie, B., and Fernandez-Pello, A. C.: Modeling transport and combustion of firebrands from burning trees, Combust. Flame, 150, 151-169, 2007.

Sardoy, N., Consalvi, J. L., Kaiss, A., Fernandez-Pello, A. C., and Porterie, B.: Numerical study of ground-level distribution of firebrands generated by line fires, Combust. Flame, 154, 478-488, 2008.
Serón, F. J., Gutiérrez, D., Magallón, J., Ferragut, L., and Asensio, M. I.: The evolution of a wildland forest fire front, Visual Comput., 21, 152-169, 2005.

Sethian, J. A. and Smereka, P.: Level set methods for fluid interfaces, Ann. Rev. Fluid Mech., 35, 341-372, 2003.

Strada, S., Mari, C., Filippi, J. B., and Bosseur, F.: Wildfire and the atmosphere: Modelling the chemical and dynamic interactions at the regional scale, Atmos. Environ., 51, 234-249, 2012.

Sullivan, A. L.: Wildland surface fire spread modelling, 1990-2007, 3: Simulation and mathematical analogue models, Int. J. Wildland Fire, 18, 387-403, 2009.

Sun, R., Jenkins, M. A., Krueger, S. K., and Charney, J.: An evaluation of fire plume properties simulated with the FDS and Clark coupled wildfire model, Can. J. Forest Res., 36, 2894-2908, 2006.

Sun, R., Krueger, S. K., Jenkins, M. A., Zuluar, M., and Charney, J. J.: The importance of fire-atmosphere coupling and boundarylayer turbulence to wildfire spread, Int. J. Wildland Fire, 18, 50 60, 2009

Viegas, D. X.: Forest fire propagation, Philos. T. Roy. Soc. Lond. A, 356, 2907-2928, 1998.

Wang, H. H.: Analysis on downwind distribution of firebrands sourced from a wildland fire, Fire Technol., 47, 321-340, 2011.

Weber, R. O., Mercer, G. N., Sidhu, H. S., and Gray, B. F.: Combustion waves for gases $(\mathrm{Le}=1)$ and solids $(\mathrm{Le} \rightarrow \infty)$, P. Roy. Soc. Lond. A, 453, 1105-1118, 1997. 